\title{
Evaluación de factores de resiliencia en niños argentinos en condiciones de vulnerabilidad familiar*
}

\section{Assessment of Resilience Factors in Argentines Children in Familiar Vulnerability Conditions}

Recibido: mayo 29 de 2013 | Revisado: abril 15 de 2014 | Aceptado: abril 15 de 2014

\author{
Gabriela Susana MORELATO** \\ Universidad del Aconcagua, Mendoza, Argentina
}

doi:10.11144/Javeriana.UPSY13-4.efrn

Para citar este artículo: Morelato, S. G. (2014). Evaluación de factores de resiliencia en niños argentinos en condiciones de vulnerabilidad familiar. Universitas Psychologica, 13(4), 1473-1488. http:// dx.doi.org/10.11144/Javeriana.UPSY13-4.efrn

* Artículo de investigación

** Instituto de Ciencias Humanas Sociales y ambientales (INCIHUSA) - CCT CONICET Mendoza. Investigadora asistente del Consejo Nacional del Investigaciones Científicas y Técnicas (CONICET). Doctora en Psicología. Docente Facultad de Psicología de la Universidad del Aconcagua, Mendoza, Argentina. Correo electrónico: gmorelato@ mendoza-conicet.gob.ar

\begin{abstract}
RESUMEN
El presente trabajo tuvo como objetivo general identificar factores asociados a la capacidad de resiliencia en una muestra de niños víctimas de maltrato infantil y analizar la relación entre variables contextuales y personales sobre la resiliencia. La muestra estuvo compuesta por 102 niños entre 6 y 13 años de edad víctimas de maltrato (físico, psicológico y negligencia). Se construyó un índice de resiliencia con base en las pruebas aplicadas a los niños y se adoptó el modelo ecológico que considera diferentes niveles contextuales en el desarrollo. Los resultados señalaron asociaciones significativas entre la resiliencia y la verbalización del problema, la presencia de baja sintomatología, el apoyo de la familia nuclear y/o extensa y la acción participativa de la comunidad.

Palabras clave resiliencia; maltrato infantil; modelo ecológico
\end{abstract}

\footnotetext{
A B S T R A C T

The purpose of this study was to assess factors associated with resilience in abused children and to analyze the relationship between context and personal variables regarding resilience. The sample consisted of 102 abused children (victims of neglect, physical abuse, and psychological abuse) between $6-13$ years-old. We built a resilience index based on tests applied to the children and we adopted the ecological model that takes into account the different context levels of development. The results point out to significant associations between resilience index and the ability to verbalize the problem, low levels of symptomatology, the support of the nuclear or extended family, and the community's active involvement.

Keywords

resilience; child abuse; ecological model
} 
El maltrato infantil es un área de estudio cuyo abordaje se torna complejo, dadas las implicancias negativas que tiene para el desarrollo de los niños. Está asociado con numerosos problemas emocionales y de conducta tales como agresión, depresión y psicopatológicos, entre otros (Cichetti \& Lynch, 1993; Cichetti \& Rogosch, 1997; Gordis, Feres, Olezeski, Rabkin, \& Trickett; 2009; Grosman \& Mesterman, 1992; Lessinger-Borges, Haag-Kristensen, \& Dalbosco-Dell Aglio, 2006; Morelato, 2011a; Moreno, 2006), ya que la problemática socava especialmente el desarrollo de la confianza básica, la sensación de seguridad y estabilidad personal. En síntesis, puede decirse que afecta el bienestar global físico y mental del niño debido a las fallas en las funciones de cuidado, permanencia y protección de sus cuidadores.

Actualmente, tanto las investigaciones pioneras acerca del maltrato infantil (Kempe \& Kempe, 1985) como investigaciones posteriores que evalúan clasificaciones (Arruabarrena \& De Paúl, 2001; Barudy, 2010; Muela, 2008) refieren la existencia de cuatro grandes categorías principales: maltrato físico, maltrato emocional o psicológico, negligencia-abandono y abuso sexual, cualquiera de las cuales afecta enormemente el desarrollo infantil. Pese a esto, en los últimos años el estudio de las problemáticas de riesgo desde el enfoque salugénico ha evolucionado y ha demostrado que, aunque el maltrato infantil determina patologías severas, existen factores que pueden moderar el desarrollo, y en consecuencia hay numerosos casos donde los individuos se desarrollan en forma esperable para su etapa evolutiva (Kotliarenco, Cáceres, \& Fontecilla, 1997; Melillo \& Suárez-Ojeda, 2001; Melillo, Suárez-Ojeda, \& Rodríguez, 2004). En este sentido, la resiliencia se entiende como una capacidad del ser humano para hacer frente a las adversidades de la vida, superarlas e inclusive ser transformado positivamente por ellas (Grotberg, 1995, 2001). Muñoz (2012), por su parte, explica que aunque ha surgido una diversidad de enfoques de la resiliencia, existe un creciente consenso en definirla como el logro de una adaptación positiva o el afrontamiento adecuado de las tareas de desarrollo típicas de una determinada etapa o cultura, a pesar de las experiencias de adversidad.

En torno al concepto y su aplicación práctica Luthar, Cicchetti y Becker (2000) hacen una revisión de la literatura científica sobre resiliencia, concluyendo con la idea de que el concepto se refiere a un dinámico proceso de adaptación dentro de un contexto de adversidad significativa. Proponen guías para operacionalizar y llevar a la práctica estudios acerca de la resiliencia, lo cual ha sido retomado por investigaciones posteriores y revisiones de tema (Luthar, 2006; Masten \& Gewirtz, 2010; Mejía, 2003; Muñoz, 2012; Naglieri \& Le Buffe, 2005; Peña, 2009; Vanistendael \& Lecomte, 2002). Desde una mirada crítica, Kalawski y Haz (2003) señalan la importancia de reconocer que la resiliencia no es en sí una variable psicológica y que su definición necesariamente dependerá del contexto en que se aplique.

En el presente estudio, se entiende la resiliencia especialmente como un proceso, producto de la interacción entre factores de riesgo y factores protectores, tratándose estos de una combinación tanto de factores individuales (tales como fortalezas, habilidades y competencias) así como contextuales relacionados con fuentes de apoyo externo (Morelato, 2011b; Rutter, 1992, 1995) que se manifiestan en la conducta y recursos personales de los niños.

Es notable que, en general, los niños maltratados tengan un desarrollo diferente y disminuido, con respecto a niños no maltratados. Por ello, se debe considerar que en el maltrato infantil los niños pueden manifestar competencia en algunas áreas y exhibir algunas dificultades en otras (Cicchetti \& Lynch, 1993). Estas áreas deben quedar definidas operacionalmente (Milling, 1998). De este modo, las investigaciones en este campo han señalado que en el ámbito del maltrato la resiliencia está vinculada a variables de naturaleza cognitiva y afectiva (Amar, Kotliarenco, \& Abello, 2003; Cicchetti, Rogosch, Lynch, \& Holt, 1993; Flores, Cichetti, \& Rogosch 2005). Asimismo, los recursos externos estarían relacionados fundamentalmente con soportes familiares y extrafamiliares, es decir, contextuales (Lazaro, 2009; Luthar et al., 2000). La influencia de los factores contextuales en el de- 
sarrollo infantil puede comprenderse mejor desde el modelo teórico de Bronfenbrenner (1981, 1987). Belsky (1993) aplicó este enfoque para explicar la etiología del maltrato infantil, teniendo en cuenta una variedad de contextos, entre los cuales se consideran los roles de los padres, las características de los hijos y los procesos que se ponen en juego entre estos, incluyendo factores asociados a la comunidad y a la cultura. Asimismo, otros autores proponen utilizar el modelo para la comprensión del proceso de resiliencia (Cassol \& De Antoni, 2006; Ehrensaft \& Tousignant, 2003). De este modo, ambos aportes se integraron sistemáticamente para el análisis de aspectos de la resiliencia en el ámbito del maltrato infantil (Figura 1; Morelato, 2011a).

En este marco, el desarrollo humano es visto como un proceso donde el niño reestructura de modo activo su ambiente y recibe el influjo de los factores vinculados con él. Está compuesto por tres núcleos interrelacionados. El primer núcleo es la persona, vista con sus características biológicas, emocionales y con aquellas constituidas por el contacto con el ambiente. El proceso o segundo núcleo es la forma en que la persona interactúa con el contexto, sus características particulares, aspectos que forman parte de su ontosistema. El tercer núcleo es el contexto, compuesto por niveles que simultáneamente van desde el contacto más íntimo del niño con los responsables de sus cuidados, hasta los contextos sociales más amplios. Tales sistemas son: microsistema, mesosistema, exosistema y macrosistema (Bronfenbrenner, 1981; Cassol \& De Antoni, 2006; Morelato, 2009, 2011a). La red familiar, las características de estos miembros y los vínculos significativos son parte del microsistema, lo cual también incluye al grupo de pares y al sistema escolar. El mesosistema se conforma por el tipo de interacción entre microsistemas, enfatizando el tipo de comunicación entre la familia y otros microsistemas cercanos (por ejemplo, la calidad de las relaciones de la familia con la escuela, con la comunidad, con la familia extensa). El exosistema se compone por aspectos relacionados a la comunicación entre las redes formales e informales, donde, si bien el niño no tiene una participación activa, indirectamente es afectado tal como la relación entre las instituciones y por las condiciones socioambientales (Shaffer, 2000). Finalmente, el macrosistema está relacionado con las políticas sociales, la cultura del país y el momento histórico.

Cuando se trata valorar en aspectos de relevancia para la resiliencia en el ámbito del maltrato infantil, se han tenido en cuenta dos dimensiones que interactúan: el riesgo y la protección (Figura 1). En el ontosistema, se destaca la relevancia de aspectos internos tales como competencias personales asociadas a aspectos académicos (Kotliarenco et al., 1997), a la fortaleza del sí mismo o autoconcepto (Cicchetti \& Rogosch, 1997; Flores et al., 2005; Kim \& Cicchetti, 2003, 2004), a las habilidades de solución de problemas (Amar et al., 2003; Muñoz, 2012; Nears, 2004) y a la creatividad entre otros (Cyrulnik, 2003; Vecina, 2006; Wolin \& Wolin, 1993). Por lo tanto, se puede pensar que las la presencia de sintomatología física o psicológica, formarían parte del ontosistema desde la dimensión de riesgo. Los problemas de conducta, de relaciones con pares u otros trastornos se incluyen también en esta dimensión, pero a nivel del microsistema. En él, las funciones de apoyo que potencian la flexibilidad, la comunicación y la cohesión son aspectos de la dimensión protectora, mientras que en la dimensión de riesgo se incluirían los indicadores específicos e inespecíficos de maltrato familiar. En el mesosistema, la función de la familia extensa (abuelos, tíos, etc.) y las buenas relaciones con la comunidad también son parte de la dimensión protectora, ya que ofrecen sostén y asistencia moral o espiritual como elemento amortiguador del riesgo (Kotliarenco et al., 1997; Moreno, 2005). La dimensión de riesgo del exosistema incluye dificultades en el acceso al empleo, a la educación o a la salud como aspectos vinculados a la vulnerabilidad social (Golovanesky, 2007). También involucra las fallas en la coordinación entre instituciones de salud, escolares, judiciales y comunitarias (Martín \& Aciego de Mendoza, 2011), acciones que forman parte del macrosistema.

En función de lo planteado, este estudio tiene como objetivo general identificar factores asociados a la capacidad de resiliencia en niños sometidos a situa- 


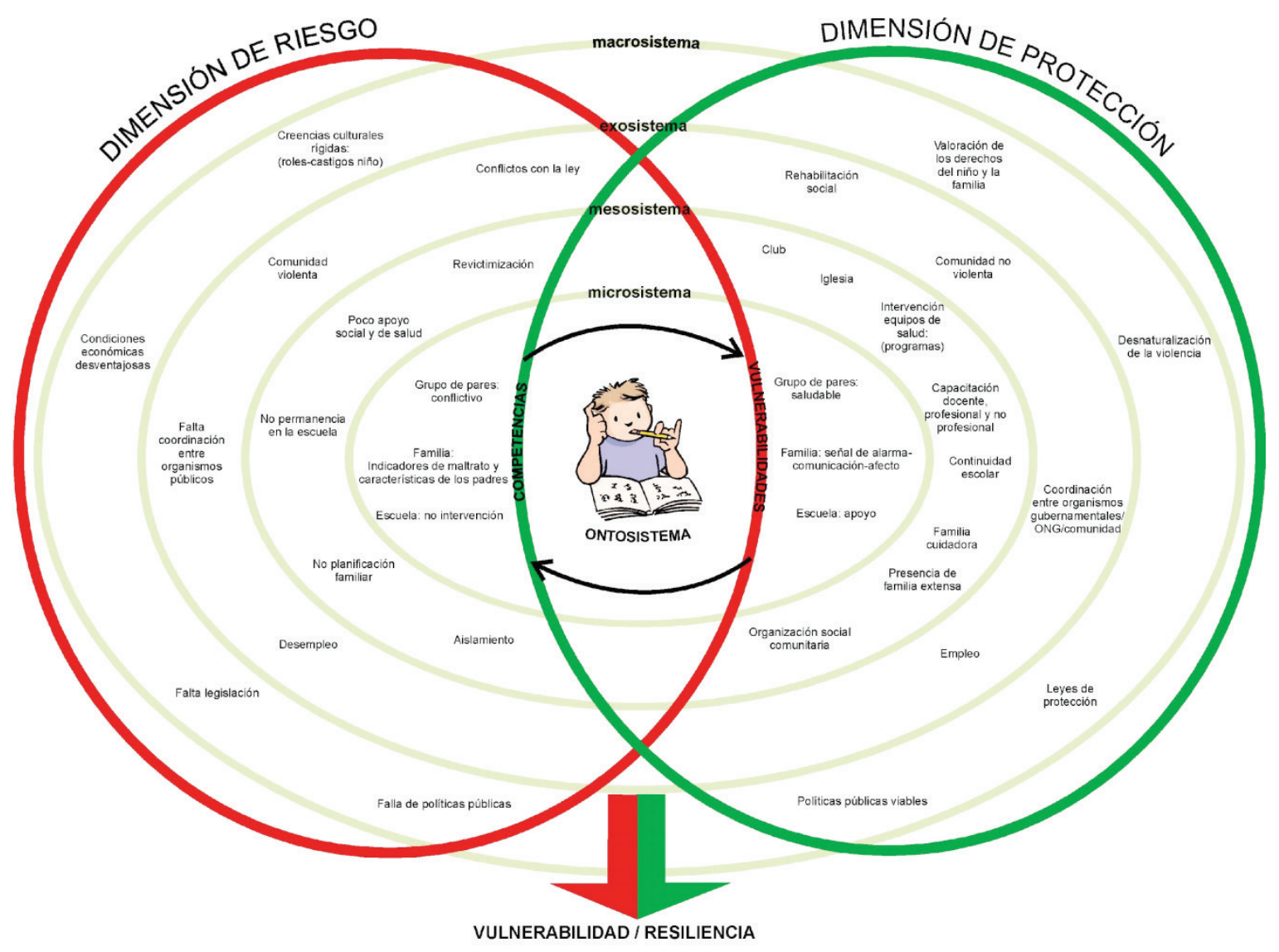

Figura 1. El proceso de resiliencia/vulnerabilidad aplicado al maltrato infantil desde el enfoque ecológico.

Fuente: Morelato (2011a)

ciones de maltrato familiar. Los objetivos específicos fueron: 1) explorar la percepción de sí mismo, de las relaciones familiares, las habilidades cognitivas para la solución de problemas interpersonales e indicadores de creatividad gráfica en los niños del grupo evaluado, 2) construir un índice de resiliencia con base en los puntajes obtenidos por los niños en las técnicas aplicadas y 3) analizar la relación entre variables contextuales y personales sobre el índice de resiliencia.

\section{Método}

El diseño de este estudio fue de tipo transversal, no experimental de enfoque mixto. Se enmarca dentro de las investigaciones de naturaleza descriptiva con características asociativas (Hernández, Fernández, \& Baptista, 2006).

\section{Participantes}

La muestra evaluada fue no probabilística de carácter intencional y ocasional. Se evaluaron a 102 niños entre 6 y 13 años de edad. El promedio de edad de la muestra total fue de 9.36 años ( $D E=$ 2.18). El $57.8 \%$ fueron varones y $42.2 \%$ niñas. Los participantes concurrían al servicio de asistencia del Programa Provincial de Atención y Prevención al Maltrato Infantil (PPMI) - Ley 6.551, dependiente del Ministerio de Salud de la provincia de Mendoza ${ }^{1}$. Los niños fueron evaluados en 9 cen-

1 Este es un programa estatal conformados 20 unidades de trabajo que funcionan en hospitales o centros de salud públicos y están constituidas por psicólogos, médicos y trabajadores sociales encargados de realizar el tratamiento, consistente en abordaje familiar durante los primeros meses y posteriormente tratamiento individual con los niños. 
tros donde funciona este programa (4 hospitales y 5 centros de salud). Todos presentaron historia de maltrato físico, maltrato emocional y/o maltrato por negligencia/abandono. No se trabajó con casos de abuso sexual, dada la complejidad para el abordaje de los mismos. El $60 \%$ de los niños fueron derivados por denuncias realizadas a una línea telefónica, destinada a recibir la información de probables situaciones de maltrato infantil. Cuando estas son confirmadas, se derivan a los equipos de salud. El $20 \%$ de los casos fueron remitidos directamente por el juzgado de familia, el $5 \%$ por derivación espontánea, es decir, que alguna persona se acercó al equipo a solicitar ayuda y el 15\% de la muestra, por otras vías (servicios de salud, escuela, etc.). Se seleccionaron casos que estaban en los inicios del tratamiento individual con los niños. Para evaluar el tipo y cantidad de indicadores de maltrato infantil, se utilizó la clasificación propuesta por Arruabarrena y De Paúl (2001). Para evaluar la severidad, se utilizó el Sistema de Clasificación de Maltrato Infantil (SCM) de Barnet, Manly y Cicchetti (1993) con la recodificación adaptada por Cicchetti, Rogosch, Manly y Lynch (2005), sumado al criterio de 5 jueces expertos locales. Como parámetro de evaluación se consideró la existencia de un $80 \%$ de acuerdo interjueces. El $89.1 \%$ de los niños presentaron indicadores de los tres tipos de maltrato en forma conjunta, es decir, combinado, el $9.8 \%$ solo maltrato psicológico y el $0.9 \%$, solo maltrato por negligencia u abandono. $\mathrm{No}$ se observó porcentaje de maltrato físico en forma aislada. La severidad se clasificó en un rango de 1 a 5 (siguiendo a los autores citados), correspondiendo el puntaje más alto al criterio de mayor severidad. El 51\% de los casos presentó un nivel de severidad media, el $24.5 \%$ severidad baja y el $24.5 \%$ restante, alta. Las familias pertenecían a sectores socialmente vulnerables valorados según lo planteado por Golovanesky (2007). Los datos fueron extraídos de las historias clínicas de los niños. Es destacable que el tipo de población que recibe este programa en su mayoría proviene de este sector, debido a que se trata de un organismo estatal de atención pública, lo cual remite a una mayor facilidad de acceder a esos datos.

\section{Instrumentos}

Análisis de historias clínicas y entrevistas con los profesionales

A fin de indagar variables socioambientales y de diagnóstico, se exploraron las historias clínicas familiares, con base en el enfoque del modelo ecológico planteado previamente. Se analizó la información de los niños mediante la técnica de análisis de contenido, contando para ello con la participación de tres colaboradores (investigadores) externos. En función de esto, se seleccionó una serie de variables ordinales de relevancia, agrupadas en categorías con base en el modelo teórico planteado previamente. Se asignó un valor 3 a los aspectos más favorables, 2 a los aspectos medianamente favorables y 1 a los aspectos desfavorables. Paralelamente, se realizó una entrevista semidirigida a los profesionales a cargo de cada caso, a los fines de completar la información y clarificar los datos seleccionados. Las variables y sus valores se detallan a continuación.

\section{Variables del ontosistema (internas)}

- Conciencia del problema en el niño (1: No reconoce problema; 2: Reconoce medianamente algunas dificultades de la interacción familiar; 3: Manifiesta y expresa el problema con claridad).

- Sintomatología (1: Presencia de sintomatología física crónica o psiquiátrica; 2: Presencia de algunos síntomas emocionales, disforias o inestabilidad; 3 : No presenta sintomatología significativa).

- Percepción del rendimiento escolar según informantes claves: docentes y/o cuidadores (1: Repitencia escolar y trastornos de aprendizaje; 2: Algunas dificultades menores de rendimiento; 3 : Buen rendimiento escolar).

\section{Variables del microsistema (relaciones más cercanas cara a cara)}

- Relaciones con pares (1: Dificultades en la relación con pares: trastornos de conducta por agresión o inhibición; 2: Dificultades menores en la relación con los pares; 3: Relaciones sociales satisfactorias). 
- Conciencia del problema en la familia nuclear (1: No toman conciencia del problema lo cual genera resistencia a las intervenciones; 2: Conciencia y colaboración en algunos miembros; 3: Conciencia y colaboración en la mayoría de los miembros de la familia).

- Miembro que requiere tratamiento especial por alcoholismo, adicciones o enfermedad psiquiátrica (1: Convive actualmente y no realiza tratamiento; 2: No convive con el niño aunque no realice tratamiento; 3: El miembro realiza tratamiento con avances conviva o no con el niño. También se incluye en este apartado cuando no hay miembros con adicciones).

- Amplitud y distribución de la vivienda (1: Hacinamiento, más de cuatro personas por cuarto; 2 : Distribución medianamente adecuada, hasta tres personas por cuarto; 3: Buena distribución, hasta dos personas por cuarto).

- Cambio y mejora en la situación de riesgo por maltrato luego del ingreso al servicio (1: Permanece con poco cambio debido al alto riesgo; 2: Se han producido modificaciones positivas con cierto alivio de la tensión familiar; 3: Se ha producido un cambio profundo que ha reestablecido el bienestar familiar).

Variables del mesosistema (relaciones entre la familia y otros grupos sociales de apoyo)

- Red familiar extensa (1: No colaboran o no hay familia extensa cercana; 2: Colaboran con ciertas reservas; 3: Ejercen funciones de apoyo y protección).

- Relación con la comunidad (1: Familia con tendencia al aislamiento, no se relacionan con la comunidad; 2: Contacto con la comunidad medianamente activo. Puede existir algún miembro de la comunidad que brinda apoyo al niño o a la familia; 3: Participan activamente en la comunidad (Iglesia, unión vecinal, instituciones recibiendo apoyo de la red social).

- Asistencia a las intervenciones de salud (esto incluye la asistencia a entrevistas familiares de acompañamiento psicoterapéutico, control de las derivaciones a otros servicios de salud o judiciales y la atención individual de los niños) (1: Grandes di- ficultades para asistir a los seguimientos; 2: Asisten con interrupciones; 3: Regularidad en la asistencia).

- Continuidad escolar (1: Deserción escolar; 2: Ausencias reiteradas a la escuela; 3: Asistencia regular).

Variables del exosistema (conexión entre las redes formales e informales)

- Situación ocupacional de padres o cuidadores (1: Desempleo; 2: Subempleo; 3: Empleo estable).

- Tipo de Comunidad (1: Comunidad con características de alto riesgo por frecuencia de acciones delictivas, altamente vulnerable socialmente; 2: Barrio o comunidad medianamente conflictiva con mejor acceso a recursos; 3 : Barrio que presenta situaciones delictivas poco frecuentes, no considerado zona de vulnerabilidad social).

- Miembro de la familia en situación de conflicto con la ley (1: Miembros en la cárcel o que han estado detenidos por delitos; 2: Miembros que han presentado algunas dificultades legales menores; 3: No ha habido en la familia conflictos con la ley).

- Apoyo y colaboración de la escuela con el equipo de salud (1: Poca o ninguna participación de la escuela, no toman contacto con la problemática de maltrato del niño; 2: Apoyo ocasional de la escuela comunicándose esporádicamente sin manifestar un compromiso claro; 3: Preocupación y colaboración de la escuela en el proceso del niño y la familia).

\section{Cuestionario de Autoconcepto}

El Cuestionario de Autoconcepto de Valdez Medina (1994) consta de 37 reactivos (adjetivos) con respuesta tipo Likert, de 5 opciones. Estos reactivos se agrupan en seis factores o dimensiones tanto reales como ideales, por lo cual es posible obtener un puntaje de autoconcepto real e ideal. Estudios empíricos señalan que las características psicométricas de este instrumento de medición son adecuadas: cuenta con un nivel de confiabilidad apropiado en niños argentinos (alpha de Cronbach $=0.82)(\mathrm{Val}$ dez Medina, 1994; Valdez Medina, Mondragón, \& Morelato, 2005). Además presenta clara validez 
de constructo (Valdez-Medina, González-Arratia, Reyes, \& Gil, 1996; Valdez-Medina, GonzálezArratia, Santos, \& Arantes, 1999, Valdez Medina et al., 2005).

Juego de las interrelaciones familiares

Este juego, elaborado por Colombo (2002, 2009), permite observar la organización y valoración que hace el niño de cada uno de los integrantes de una familia: los personajes más queridos, más denigrados y aspectos relacionados con la interacción familiar. No es una técnica psicométrica, sino un instrumento cualitativo formado por una serie de tarjetas con dibujos de personas (adultos, ancianos, niños), cuya consigna consiste en solicitar al niño que arme una familia. Desde el punto de vista de la validez de constructo, según su autora, el juego fue creado para ser utilizado en la clínica de niños víctimas de maltrato, ya que funciona como disparador de situaciones vividas en la propia familia, abre posibilidades de diálogo y suscita la evocación de la experiencia familiar. Dada su estructura, se analizó la frecuencia de aparición y el tipo de respuestas dadas por los niños, se conformaron variables dicotómicas de análisis y se elaboraron definiciones operacionales para cada una de ellas. Las variables observadas se agruparon en dos indicadores (Colombo, Morelato, \& Gurvich, 2009):

- Indicadores protectores de la percepción de la familia: conformado por la percepción de una serie de aspectos positivos de la familia (concepto adecuado de familia; mención de personajes valorados; identificación con un personaje valorado; expresión de roles y/o algún tipo de conflicto).

- Indicadores de riesgo de la percepción de familia: conformado por la percepción de una serie de aspectos negativos de la familia (concepto inadecuado o incompleto de familia; mención de personajes desvalorizados incluyendo el propio niño; identificación con un personaje desvalorizado; construcción de una familia confusa).
Test de Evaluación de Habilidades

Cognitivas para la Solución de Problemas

Interpersonales (EVHACOSPI)

El Evhacospi (García \& Magaz, 1998) es una técnica creada con la finalidad de evaluar de manera cuantitativa y cualitativa las destrezas cognitivas relacionadas con los procesos de solución de problemas interpersonales y que permiten conocer la amplitud y flexibilidad del pensamiento infantil. Analiza variables tales como: identificación de situación problema; descripción del problema; identificación de la emoción involucrada; identificación de clase y cantidad de emociones, generación de alternativas de diversos tipos, anticipación de consecuencias y toma de decisiones (Morelato, Maddio, \& Ison 2006). La técnica cuenta con validez de constructo fundamentada sobre la base de los estudios de las habilidades cognitivas presentes en el proceso de solución de problemas interpersonales (Spivack, Platt, \& Shure, 1976).

\section{Test de Creatividad Gráfica}

Para evaluar la creatividad se utilizó el Test de Creatividad Gráfica de Marín Ibáñez (1995). Esta técnica consta de 35 estímulos y permite explorar la creatividad, a partir de una prueba gráfica que indaga principalmente tres indicadores: 1) Fluidez: entendida como la capacidad de realizar la mayor cantidad de respuestas posibles sin repetir el patrón; 2) Flexibilidad: es decir, la habilidad para generar y pensar distintas clases de soluciones y 3) Originalidad: o destreza para dar respuestas inusuales, diferentes a las demás o poco frecuentes. Su validez de contenido está basada en investigaciones de Torrance y Miers (1970) y también en los trabajos de Guilford (1950).

\section{Procedimiento}

En primer lugar, se solicitó la autorización para llevar a cabo el proyecto a los directivos del PPMI. Luego de obtener la autorización de esta entidad se solicitó la misma al comité de ética que funciona en los hospitales o centros de salud donde se trabajó. 
Posteriormente, se contactó a los profesionales de los equipos, a fin de explicar los objetivos de trabajo y obtener su colaboración. Previamente a la evaluación de los niños en entrevistas preliminares, los psicólogos a cargo de cada caso explicaron el propósito del estudio y el procedimiento a los padres o tutores, solicitando su consentimiento informado y asegurando la confidencialidad de los datos y su participación voluntaria. Asimismo, a los niños también se les explicó el sentido de trabajar con ellos, haciendo énfasis también en la voluntariedad, aspecto avalado actualmente por la Ley 26 061 (art. 24) de protección integral de los derechos del niño. Se realizaron dos o tres entrevistas con cada niño, efectuadas por un entrevistador entrenado en presencia de los profesionales a cargo, en los casos de mayor gravedad y/o cuando el profesional lo consideraba pertinente por las características del niño. La administración fue en forma individual. Los casos fueron seleccionados por los profesionales a cargo y por el investigador, según los criterios previamente previstos. Con los casos seleccionados que en momento de evaluación presentaban algún tipo de crisis, por ejemplo, una nueva situación de violencia, se suspendía el trabajo para dar lugar a intervenciones terapéuticas adecuadas. En otro momento, y si era posible, se completaba la evaluación según la evolución emocional del niño y la familia, o si no podía completarse en más del $70 \%$ de las técnicas, se descartaban.

\section{Tratamiento estadístico}

El análisis estadístico de los resultados se efectuó utilizando procedimientos principalmente no paramétricos, por medio del programa estadístico Statistical Package for Social Sciences (SPSS) versión 17.0. Se realizó un análisis bivariado, mediante la prueba chi cuadrado y coeficiente Tau-b de Kendall para variables de nivel de medición ordinal. El nivel de significación empleado fue de $\alpha=0.05$.

\section{Construcción del índice de resiliencia}

Para construir y definir un parámetro de competencia en función de la resiliencia en el ámbito del maltrato (Milling, 1998), se siguieron los criterios de varios autores (Cicchetti et al., 1993; Flores et al., 2000; Luthar et al., 2000). Se utilizaron ocho indicadores de competencia que correspondieron a variables que, de acuerdo al criterio teórico planteado al inicio de este trabajo, estuvieron relacionadas con el proceso de resiliencia y además se asociaron entre sí significativamente, desde el punto de vista estadístico. Se hallaron relaciones significativas entre la generación de alternativas, variable que forma parte del índice y las siguientes variables: autoconcepto real $\left(\chi^{2}=17.4, p<0.002\right)$, emociones mencionadas por el niño $\left(\chi^{2}=13.16, p<0.003\right)$, anticipación de consecuencias $\left(\chi^{2}=45.52, p<\right.$ 0.001 ), indicadores protectores de la percepción de la familia $\left(\chi^{2}=12.48, p<0.01\right)$ y los tres indicadores de creatividad: fluidez $\left(\chi^{2}=23.76, p<0.001\right)$, flexibilidad $\left(\chi^{2}=17.4, p<0.002\right)$ y originalidad $\left(\chi^{2}=27.05, p<0.001\right)$. Estas asociaciones indican que los niños con mejor capacidad para generar alternativas asertivas también pudieron anticipar consecuencias, mencionar emociones y obtuvieron buenos puntajes en su autoconcepto, en la creatividad y en la percepción de indicadores protectores de las interrelaciones familiares. Con posterioridad, se estandarizaron los puntajes de cada una de las variables asociadas entre sí, utilizando las puntuaciones $\mathrm{Z}$ a fin de asegurar la comparabilidad entre las mismas (Aron \& Aron, 2001) y se establecieron rangos resultando que un $36.3 \%$ de niños obtuvieron un rango de resiliencia alto, el $39.2 \%$ medio y el $24.53 \%$ bajo.

\section{Resultados}

Las tablas de contingencia que se exponen en este trabajo describen las asociaciones significativas halladas entre el índice de resiliencia y las variables contextuales que resultaron significativas.

En la Tabla 1 se observa, teniendo en cuenta que todos los niños han sido víctimas de diversos tipos de situaciones de maltrato, que el $80 \%$ de los niños de rango de resiliencia bajo no expresan la existencia de algún tipo de problema en la familia. En contrapartida, el $70.3 \%$ de los niños de rango alto expresan que existe o ha existido alguna dificultad 


\section{TABLA 1}

Prueba de $\chi^{2}$ entre el índice de resiliencia y la conciencia del problema del niño de la situación de maltrato

\begin{tabular}{lcccc}
\hline \multirow{2}{*}{ Índice de resiliencia } & \multicolumn{4}{c}{ Conciencia del problema en el niño } \\
\cline { 2 - 5 } & $\begin{array}{c}\text { No reconoce } \\
\text { problema (\%) }\end{array}$ & $\begin{array}{c}\text { Reconoce medianamente } \\
\text { dificultades (\%) }\end{array}$ & $\begin{array}{c}\text { Reconoce y expresa } \\
\text { problema (\%) }\end{array}$ & $\begin{array}{c}\text { Total } \\
(\%)\end{array}$ \\
\hline Bajo (25) & 80 & 16 & 4 & 100 \\
Medio (40) & 42 & 32.5 & 25 & 100 \\
Alto (37) & 0 & 29.7 & 70.3 & 100 \\
\hline Total (102) & 36.3 & 27.5 & 36.3 & 100 \\
\hline
\end{tabular}

$\chi^{2}=49.03, p<0.0001$, Tau $-b=0.611$.

Fuente: elaboración propia

TABLA 2

Prueba de $\chi^{2}$ entre el índice de resiliencia y presencia de sintomatología en el niño

\begin{tabular}{ccccc}
\hline \multirow{2}{*}{ Índice de resiliencia } & \multicolumn{4}{c}{ Presencia de sintomatología } \\
\cline { 2 - 5 } & $\begin{array}{c}\text { Sintomatología } \\
\text { psiquiátrica o física (\%) }\end{array}$ & $\begin{array}{c}\text { Algunos síntomas } \\
\text { emocionales (\%) }\end{array}$ & $\begin{array}{c}\text { No presenta } \\
\text { sintomatología (\%) }\end{array}$ & $\begin{array}{c}\text { Total } \\
(\%)\end{array}$ \\
\hline Bajo (25) & 40 & 36 & 24 & 100 \\
Medio (40) & 22.5 & 45 & 32.5 & 100 \\
Alto (37) & 13.5 & 8.1 & 78.4 & 100 \\
\hline Total (102) & 23.5 & 29.4 & 47.1 & 100 \\
\hline
\end{tabular}

$\chi^{2}=26.2, p<0.0001$, Tau-b $=0.384$.

Fuente: elaboración propia

TABLA 3

Prueba de $\chi^{2}$ entre índice de resiliencia y la percepción del rendimiento escolar según informantes claves

\begin{tabular}{ccccc}
\hline \multirow{2}{*}{ Índice de resiliencia } & \multicolumn{4}{c}{ Percepción del Rendimiento Escolar } \\
\cline { 2 - 5 } & $\begin{array}{c}\text { Repitencia escolar y/o } \\
\text { trastornos de aprendizaje (\%) }\end{array}$ & $\begin{array}{c}\text { Algunas dificultades menores } \\
\text { en el rendimiento (\%) }\end{array}$ & $\begin{array}{c}\text { Buen rendimiento } \\
\text { escolar (\%) }\end{array}$ & $\begin{array}{c}\text { Total } \\
(\%)\end{array}$ \\
\hline Bajo (25) & 56 & 24 & 20 & 100 \\
Medio (40) & 30 & 42.5 & 27.5 & 100 \\
Alto (37) & 16.2 & 8.1 & 75.7 & 100 \\
\hline Total (102) & 31.4 & 25.5 & 43.1 & 100 \\
\hline
\end{tabular}

$\chi^{2}=30.96, p<0.0001$ Tau-b $=0.415$.

Fuente: elaboración propia

en su familia, lo cual los ha llevado a la consulta al servicio. Asimismo, en la Tabla 2 se presenta la relación existente entre el índice y la presencia de algún tipo de sintomatología en el niño, donde se observa que el $78.4 \%$ de los niños de alto rango de resiliencia no presentaron sintomatología física o psiquiátrica significativa. Asimismo, la Tabla 3 indicó la relación entre la percepción del rendimiento escolar y el índice de resiliencia, destacándose según lo valorado por los docentes, que el 75.7\% de los niños de alto rango de resiliencia presentó un buen rendimiento escolar. A la inversa, el 56\% de los niños de rango bajo presenta trastornos de aprendizaje y dificultades escolares.

Desde la perspectiva del microsistema, se observó una clara asociación entre los niños de resiliencia alta y relaciones sociales satisfactorias entre pares. La Tabla 4 señala que el $51.4 \%$ de los niños rango 
TABLA 4

Prueba de $\chi^{2}$ entre índice de resiliencia y relación con los pares

\begin{tabular}{ccccc}
\hline \multirow{2}{*}{ Índice de resiliencia } & \multicolumn{4}{c}{ Relaciones con pares } \\
\cline { 2 - 5 } & $\begin{array}{c}\text { Trastornos de conducta } \\
\text { (agresión o inhibición) }\end{array}$ & $\begin{array}{c}\text { Relaciones } \\
\text { medianamente adec. (\%) }\end{array}$ & $\begin{array}{c}\text { Buenas relaciones } \\
\text { con pares (\%) }\end{array}$ & $\begin{array}{c}\text { Total } \\
(\%)\end{array}$ \\
\hline Bajo (25) & 36 & 52 & 12 & 100 \\
Medio (40) & 32.5 & 42.5 & 25 & 100 \\
Alto (37) & 13.5 & 35.1 & 51.4 & 100 \\
\hline Total (102) & 26.5 & 42.2 & 31.4 & 100 \\
\hline
\end{tabular}

$\chi^{2}=12.97, p<0.011$, Tau-b $=0.295$.

Fuente: elaboración propia

TABLA 5

Prueba de $\chi^{2}$ entre índice de resiliencia y conciencia del problema desde la percepción de los miembros de la familia

\begin{tabular}{ccccc}
\hline \multirow{2}{*}{ Índice de resiliencia } & \multicolumn{4}{c}{ Conciencia del problema en la familia nuclear } \\
\cline { 2 - 5 } & $\begin{array}{c}\text { No conciencia del } \\
\text { problema (\%) }\end{array}$ & $\begin{array}{c}\text { Conciencia en algún } \\
\text { miembro de la flía. (\%) }\end{array}$ & $\begin{array}{c}\text { Colaboración y reflexión de } \\
\text { varios miembros flía. (\%) }\end{array}$ & $\begin{array}{c}\text { Total } \\
(\%)\end{array}$ \\
\hline Bajo (25) & 76 & 24 & 0 & 100 \\
Medio (40) & 47.5 & 50 & 2.5 & 100 \\
Alto (37) & 18.9 & 64.9 & 16.2 & 100 \\
\hline Total (102) & 44.1 & 49 & 6.9 & 100 \\
\hline
\end{tabular}

$\chi^{2}=23.82, p<0.0001$, Tau-b $=0.436$.

Fuente: elaboración propia

de resiliencia alto presenta buenas relaciones con sus compañeros y solo el $12 \%$ de los niños de rango bajo, manifiesta estar a gusto en las relaciones con sus compañeros o amigos. Por otra parte, en la Tabla 5 se observa que la presencia de algún miembro de la familia que reconoce las dificultades o puede reflexionar sobre ellas se asoció con un alto rango de resiliencia (64.9\%).

En cuanto al mesosistema, fue observable que el índice de resiliencia alto se relacionó con la mejora en la situación familiar y con la regularidad en la asistencia a los servicios de salud, mientras que la permanencia de la situación de maltrato y la deserción en la asistencia al tratamiento estuvo asociada a la resiliencia baja.

Los datos más significativos de la Tabla 7 indican que el 64\% de los niños con bajo índice de resiliencia posee una familia extensa que no interviene como fuente de apoyo externo. Asimismo, la Tabla 8 muestra que el $56 \%$ de niños de rango

TABLA 6

Prueba de $\chi^{2}$ entre índice de resiliencia y la evolución de la situación de maltrato

\begin{tabular}{ccccc}
\hline & \multicolumn{4}{c}{ Situación de maltrato } \\
\cline { 2 - 5 } Índice de resiliencia & $\begin{array}{c}\text { Permanece presente } \\
\text { actualmente (\%) }\end{array}$ & $\begin{array}{c}\text { Cierto cambio y alivio } \\
\text { de la tensión (\%) }\end{array}$ & $\begin{array}{c}\text { Cambio y } \\
\text { bienestar (\%) }\end{array}$ & $\begin{array}{c}\text { Total } \\
(\%)\end{array}$ \\
\hline Bajo (25) & 56 & 36 & 8 & 100 \\
Medio (40) & 32.5 & 45 & 22.5 & 100 \\
Alto (37) & 10.8 & 51.4 & 37.8 & 100 \\
\hline Total (102) & 30.4 & 45.1 & 24.5 & 100 \\
\hline
\end{tabular}

$\chi^{2}=16.42, p<0.002$, Tau-b $=0.352$.

Fuente: elaboración propia 


\section{TABLA 7}

Prueba de $\chi^{2}$ entre índice de resiliencia y presencia de la colaboración de la familia extensa

\begin{tabular}{ccccc}
\hline \multirow{2}{*}{ Índice de resiliencia } & \multicolumn{4}{c}{ Familia extensa } \\
\cline { 2 - 5 } & $\begin{array}{c}\text { No tiene familia extensa o } \\
\text { esta no interviene (\%) }\end{array}$ & $\begin{array}{c}\text { Colaboran con ciertas } \\
\text { reservas (\%) }\end{array}$ & $\begin{array}{c}\text { Familia extensa } \\
\text { protectora (\%) }\end{array}$ & $\begin{array}{c}\text { Total } \\
(\%)\end{array}$ \\
\hline Bajo (25) & 64 & 4 & 32 & 100 \\
Medio (40) & 65 & 15 & 20 & 100 \\
Alto (37) & 37.8 & 35.1 & 27 & 100 \\
\hline Total (102) & 54.9 & 19.6 & 25.5 & 100 \\
\hline
\end{tabular}

$\chi^{2}=12.09, p<0.017$, Tau-b $=0.14$

Fuente: elaboración propia

\section{TABLA 8}

Prueba de $c^{2}$ entre índice de resiliencia y relaciones de la familia con la comunidad

\begin{tabular}{ccccc}
\hline \multirow{2}{*}{ Índice de resiliencia } & \multicolumn{4}{c}{ Relación con la comunidad de la familia } \\
\cline { 2 - 5 } & $\begin{array}{c}\text { Tendencia al } \\
\text { aislamiento (\%) }\end{array}$ & $\begin{array}{c}\text { Escasos contactos con la } \\
\text { comunidad (\%) }\end{array}$ & $\begin{array}{c}\text { Participación activa } \\
\text { (iglesia, club, etc.) }\end{array}$ & $\begin{array}{c}\text { Total } \\
(\%)\end{array}$ \\
\hline Bajo (25) & 56 & 28 & 16 & 100 \\
Medio (40) & 30 & 50 & 20 & 100 \\
Alto (37) & 5.4 & 56.8 & 37.8 & 100 \\
\hline Total (102) & 27.5 & 47.1 & 25.5 & 100 \\
\hline
\end{tabular}

$\chi^{2}=20.38, \quad p<0.0001$, Tau-b $=0.355$.

Fuente: elaboración propia

\section{TABLA 9}

Prueba de $\chi^{2}$ entre índice de resiliencia y la continuidad en la asistencia a los equipos de salud

\begin{tabular}{ccccc}
\hline \multirow{2}{*}{ Índice de resiliencia } & \multicolumn{4}{c}{ Asistencia a equipos de salud } \\
\cline { 2 - 5 } & $\begin{array}{c}\text { Asistencia a equipos de } \\
\text { salud (\%) }\end{array}$ & $\begin{array}{c}\text { Asistencia a equipos de } \\
\text { salud (\%) }\end{array}$ & $\begin{array}{c}\text { Asistencia a equipos } \\
\text { de salud (\%) }\end{array}$ & $\begin{array}{c}\text { Total } \\
(\%)\end{array}$ \\
\hline Bajo (25) & 16 & 60 & 24 & 100 \\
Medio (40) & 17.5 & 37.5 & 45 & 100 \\
Alto (37) & 0 & 29.79 & 70.3 & 100 \\
\hline Total (102) & 10.8 & 40.2 & 49 & 100 \\
\hline
\end{tabular}

$\chi^{2}=16.54, p<0.002$, Tau-b $=0.338$.

Fuente: elaboración propia

\section{TABLA 10}

Prueba de $\chi^{2}$ entre índice de resiliencia y continuidad escolar

\begin{tabular}{ccccc}
\hline \multirow{2}{*}{ Índice de resiliencia } & \multicolumn{4}{c}{ Continuidad escolar } \\
\cline { 2 - 5 } & $\begin{array}{c}\text { Deserción escolar } \\
(\%)\end{array}$ & $\begin{array}{c}\text { Ausencias reiteradas } \\
(\%)\end{array}$ & $\begin{array}{c}\text { Asistencia adecuada } \\
(\%)\end{array}$ & $\begin{array}{c}\text { Total } \\
(\%)\end{array}$ \\
\hline Bajo (25) & 4 & 44 & 52 & 100 \\
Medio (40) & 7.5 & 10 & 82.5 & 100 \\
Alto (37) & 0 & 10.8 & 89.2 & 100 \\
\hline Total (102) & 3.9 & 18.6 & 77.5 & 100 \\
\hline
\end{tabular}

$\chi^{2}=17.09, p<0.002$, Tau-b $=0.29$.

Fuente: elaboración propia 
de resiliencia bajo proviene de familias que tienen poco contacto con la comunidad y que tienden al aislamiento. En cambio, el 56.8\% de los niños con alto índice de resiliencia forman parte de familias que tienen algún contacto con las instituciones comunitarias y el $37.8 \%$ participa activamente de las mismas.

En lo que respecta a la asistencia al seguimiento de salud en los servicios especializados en la temática del maltrato infantil, se observó que el $70.3 \%$ de niños de rango de resiliencia alto asiste con alguien de su familia a los controles y seguimientos del servicio en forma regular, mientras que en el 60\% de las familias, los niños de rango bajo presenta dificultades para asistir. Finalmente, en relación con la asistencia escolar, la Tabla 10 señala que el $89.2 \%$ de los menores que tuvieron índice de resiliencia alto asiste a la escuela en forma regular y que ninguno de ellos presenta deserción escolar. Por otra parte, el $44 \%$ de niños de rango bajo presenta ausencias reiteradas a la escuela. Para el caso del exosistema (el cual se relacionó con las situaciones de empleo, el conflicto con la ley y con el tipo de comunidad), no se halló asociación con el índice de resiliencia. Sería importante profundizar el estudio de estos aspectos en posteriores investigaciones a fin de corroborar o no estos hallazgos.

\section{Discusión}

Respecto del ontosistema (Tablas 1, 2 y 3) se observó que un gran porcentaje de niños de rango de resiliencia bajo no expresan la existencia de algún tipo de problema en la familia vinculada a la razón por la cual han ingresado al servicio de salud. Pollack (2006) plantea que en las investigaciones actuales un aspecto de la resiliencia fundamental se relaciona con la capacidad de sentir, experienciar y ser libre de expresar vulnerabilidad, dentro de un contexto de conexiones conformadas por cuidadores adultos. Por ello, es interesante observar que un buen porcentaje de niños de rango alto reconocen y expresan que existe alguna dificultad en su familia, lo cual puede considerarse un recurso de salud. Asimismo, se manifestó una relación entre la resiliencia baja y el aumento de sintomatología.
Esto nos corrobora la importancia de observar en primera instancia y como indicador de buen pronóstico, la capacidad infantil de poner en palabras aquellas dificultades familiares que les generan sufrimiento. De ahí que, es probable que aquellos niños que hayan podido expresar el problema tengan mayor capacidad de simbolizar con palabras sus dificultades, manifiesten menos sintomatología psiquiátrica o física crónica (Tabla 2) y presenten menos trastornos de aprendizaje y dificultades escolares (Tabla 3).

Desde la perspectiva del microsistema, se observó una clara asociación entre los niños de rango alto con las relaciones sociales satisfactorias entre pares (Tabla 4) y la presencia de algún miembro de la familia que reconocía las dificultades o podía reflexionar sobre ellas (Tabla 5). Esto indica que los niños más fortalecidos en sus recursos internos contaban dentro de su familia nuclear algún miembro que tomaba conciencia del problema y ponía en marcha recursos protectores, tales como denunciar o pedir ayuda por ejemplo. En contrapartida, los niños de rango de resiliencia bajo se asociaron con familias que mostraron resistencia a las intervenciones, ya que sus miembros no reconocían ninguna dificultad. Por ende, la resiliencia también estaría asociada con el entorno de relaciones cercanas, especialmente con la familia, los pares y los adultos significativos (Lázaro, 2009; Luthar et al., 2000). Del mismo modo, en el mesosistema, fue observable que el índice de resiliencia alto se relacionó con la mejora en la situación de tensión familiar (Tabla 6), lo cual probablemente corresponda con otra de las asociaciones que fue la regularidad en la asistencia a los servicios de salud (Tabla 9), mientras que la permanencia de la situación de maltrato estuvo vinculada al rango de resiliencia bajo.

Por lo tanto, y para sintetizar, se puede pensar que la presencia de alguien que logra comprender que existe un problema grave en la familia, que pide ayuda y colabora, permite dar continuidad al seguimiento de salud y aliviar las tensiones, lo cual incide positivamente en el desarrollo de recursos. En las Tablas 7 y 8 se manifiesta la importancia de las fuentes de apoyo externo vinculadas a la familia extensa y a la comunidad escolar para el desarrollo 
de la resiliencia. Ambos aspectos se pueden relacionar con lo planteado por Moreno (2005), quien señala que uno de los factores que se ha identificado con mayor frecuencia en las revisiones teóricas y empíricas es el aislamiento social, como una de las causas del maltrato infantil. Asimismo, indica que en ciertos tipos de maltrato, como la negligencia o abandono, también se observa gran conflicto con la familia extensa, lo cual hace que los cuidadores no recurran a la misma en busca de apoyo o de ayuda, ya que las respuestas generalmente son negativas.

De los hallazgos de la presente investigación se puede afirmar que el aislamiento social, tanto desde lo comunitario como desde lo familiar, no solo está vinculado al maltrato, sino también a los bajos niveles de resiliencia en los niños. Esto probablemente esté relacionado con la función de la red social, la cual desempeña tareas básicas para la supervivencia de sus miembros, ya que mejora la calidad de vida de sus integrantes y también mantiene el vínculo social (Abello \& Madariaga, 1999; Afifi \& McMillan, 2011; Henderson \& Milstein, 2003).

Además, se ha observado que la presencia de intereses y vínculos afectivos con personas significativas fuera del contexto familiar, favorece la manifestación de comportamientos resilientes en circunstancias familiares adversas, convirtiéndose esta red social en un factor protector cuando los menores llegan a la edad adulta (Lázaro, 2009). En lo que respecta a la asistencia familiar al seguimiento de salud en los servicios especializados de asistencia al maltrato infantil, también se observó una asociación significativa con el rango de resiliencia alto, así como con la asistencia escolar. Estos hallazgos ratifican la importancia de las instituciones como redes sociales de apoyo para el desarrollo de recursos.

\section{Conclusiones y limitaciones}

El estudio presentado pudo describir la asociación existente una serie de factores sociocognitivos y afectivos. Esto permitió construir un índice vinculado a la potencial capacidad interna de los niños para superar y sobreponerse de las situaciones de riesgo sufridas. En esta línea, se observaron asociaciones entre este índice y factores contextuales. Con base en lo planteado, puede decirse que, frente a las situaciones de maltrato infantil, es posible estimular la puesta en marcha de procesos de resiliencia, si se fomentan potencialidades y recursos vinculados, por un lado, a las habilidades internas (autoconcepto, creatividad, habilidades de solución de problemas, percepción de aspectos positivos de la familia) y, por otra parte, a la dimensión de protección del contexto. Estos aspectos se asocian a la expresión de la problemática, a los vínculos significativos con pares, a la toma de conciencia del problema en la familia nuclear, al apoyo de la familia extensa, de la comunidad y a la permanencia en el sistema escolar y de salud. Es probable que al tener en cuenta estos factores también sea posible potenciar el desarrollo de la resiliencia del niño y la familia. Asimismo, en contextos de alto riesgo, sería de gran utilidad la implementación en la escuela y la comunidad de programas de intervención que fortalezcan estos factores desde en un enfoque preventivo, lo cual parece dar buenos resultados (Acevedo \& Mondragón, 2005; Masten \& Gewirts, 2010).

Para finalizar, se plantea que sería sumamente enriquecedor replicar y profundizar estos estudios en el ámbito del maltrato infantil. Abordar el maltrato infantil desde la perspectiva de la resiliencia implica generar un espacio para que los profesionales de la salud puedan formar parte del contexto infantil, brindando guías del desarrollo (Cyrulnik, 2003), lo cual implica ofrecer recursos de apoyo externos que permitan sostener el proceso investigado.

En este estudio, se consideró como limitación el hecho de que al trabajar con muestras clínicas se incrementa la dificultad para aumentar la cantidad de casos, dadas las características de la problemática y las dificultades de acceso a las mismas, fundamentalmente por la necesidad de preservar a los niños. Por lo tanto, sería sumamente útil replicar estudios que tengan en cuenta los aspectos de contexto aquí trabajados, a los fines de fortalecer o discutir los resultados que se presentan.

\section{Referencias}

Amar, J. J., Kotliarenco, M., \& Abello, R. (2003). Factores psicosociales asociados con la resiliencia en 
niños colombianos víctimas de violencia intrafamiliar. Investigación y Desarrollo, 11(1), 162-197.

Abello, R., \& Madariaga, C. (1999). Las redes sociales ipara qué? Psicología desde el Caribe, 2(3), 116-135.

Acevedo, V., \& Mondragón, H. (2005). Resilencia y escuela. Pensamiento Psicológico, 1(5), 21-35.

Afifi, T., \& McMillan, H. L. (2011). Resilience following child maltreatment: A review of protective factors. Revue Canadienne de Psychiatrie, 56(5), 266-272.

Argentina, Mendoza. Ley Provincial 6551 de 1997. Creación del Programa Provincial de Niñez y Adolescencia. Diciembre 10 de 1997. Decreto reglamentario 2107 de 1998 - B. O.

Argentina. Ley Nacional 26061 de 2005. Protección Integral de los Derechos de Niñas, Niños y Adolescentes. Octubre 26 de 2005. B. O. №30767.

Aron, A., \& Aron, E. (2001). Estadística para psicología (2.a ed.). Buenos Aires: Prentice Hall.

Arruabarrena, M., \& De Paúl (2001). Maltrato a los niños en la familia. Evaluación y tratamiento (6.a ed.). Madrid: Pirámide.

Barnet, D., Manly, J., \& Cicchetti, D. (1993). Defining maltreatment: The interface between policy and research. En D. Cicchetti \& S. Toth (Eds.), Advances in applied developmental psychology: Child abuse, child development and social policy (Vol. 8, pp. 7-73). New Jersey: Ablex Publishing Corporation.

Barudy, J. (1998). El dolor invisible de la infancia. Barcelona: Paidós.

Belsky, J. (1993). Etiology of child maltreatment: A developmental-ecological analysis. Psychological Bulletin, 114(3), 413-434.

Bronfenbrenner, U. (1981). The ecology of human development. Experiments by nature and design. Cambridge, MA: Harvard University Press.

Bronfenbrenner, U. (1987). La ecología el desarrollo humano: experimentos en entornos naturales y diseñados. Barcelona: Paidós.

Cassol, L., \& De Antoni, C. (2006). Família e abrigo como rede de apoio social e afetiva. En D. Dalbosco Dell `Aglio, S. H. Koller \& M. A. Mattar Yunes (Orgs.), Resiliência e psicología positiva: interfaces do risco à proteção (pp. 173-201). São Paulo: Casa do Psicólogo.
Cicchetti, D., \& Lynch, M. (1993). Toward an ecological/ transactional model of community violence and child maltreatment: Consequences for children's development. Psychiatry. Interpersonal \& Biological Processes, 56(1), 96-117.

Cicchetti, D., \& Rogosch, F. (1997). The role of self organization en the promotion of resilience in maltreated children. Development and Psychopathology, 9(4), 797-815.

Cicchetti, D., Rogosch, F., Lynch, M., \& Holt, K. (1993). Resilience in maltreated children: Processes leading to adaptive outcome. Development and Psychopathology, 5(4), 629-647.

Cicchetti, D., Rogosch, F., Manly, J. T., \& Lynch, M. (2005). Longitudinal pathways to resilience in maltreated children. User guide and codebook. [Dataset]. National Data Archive on Child Abuse and Neglect. Family Life Development Center, Cornell University, Ithaca, New York. Disponible en http:// www.ndacan.cornell.edu

Colombo, M. (2002). Juego de las interrelaciones familiares. Buenos Aires: Cauquén.

Colombo, R. (2009). El juego de interrelaciones familiares. Aplicación en clínica y en evaluación. Buenos Aires: Cauquén.

Colombo, R., Morelato, G., \& Gurvich, M. (2009). El juego de interrelaciones familiares: aportes para la sistematización de su evaluación desde un estudio realizado en la provincia de Mendoza. En R. Colombo (Comp.), El juego de interrelaciones familiares. Aplicación en clínica y en evaluación (pp. 63-69). Buenos Aires: Cauquén.

Cyrulnik, B. (2003). Los patitos feos. La resiliencia: una infancia infeliz no determina la vida. Barcelona: Gedisa.

Ehrensaft, E., \& Tousignant, M. (2003). Ecología humana y social de la resiliencia. En M. Manciaux (Comp.), La resiliencia: resistir y rehacerse (pp. 159. 174). Barcelona: Gedisa.

Flores, E., Cicchetti, D., \& Rogosch, F. (2005). Predictors of resilience in maltreated and nonmaltreated Latino children. Developmental Psychology, 41(2), 338-351.

García, J., \& Magaz, A. (1998). Evhacospi. Test de Evaluación de Habilidades Cognitivas para la Solución 
de Problemas Interpersonales. Manual de referencia. Madrid: Grupo ALBOR-COHS.

Grotberg, E. (1995). The International Resilience Project:

Promoting resilience in children (Informe técnico N. ㄴ 143). Birmingham, AL: Civitan International Research Center. (ERIC ED 383 424)

Grotberg, E. (2001). Nuevas tendencias en resiliencia. En A. Melillo \& N. Suárez Ojeda (Comps.), Resiliencia: descubriendo las propias fortalezas (pp. 19-30). Buenos Aires: Paidós.

Golovanesky, L. (2007). Vulnerabilidad social: una propuesta para su medición en Argentina. Revista de Economía y Estadística, 45(2), 53-94.

Gordis, E., Feres, N., Olezeski, C., Rabkin, A., \& Trickett, P. (2010). Skin conductance reactivity and respiratory sinus arrhythmia among maltreated and comparison youth: Relations with aggressive behavior. Journal of Pediatric Psychology, 35(5), 547 558. http://dx.doi.org/10.1093/jpepsy/jsp113

Grosman, C., \& Mesterman, S. (1992). Maltrato al menor: el lado oculto de la escena familiar. Buenos Aires: Editorial Universidad.

Guilford, J. P. (1950). Creativity. American Psychologist, 5(9), 444-454.

Henderson, N., \& Milstein, M. (2003). Resiliencia en la escuela. Buenos Aires: Paidós.

Hernández, R., Fernández, C., \& Baptista, P. (2006). Metodología de la investigación (4.a ed.). México: Mc Graw Hill.

Kalawski, J. P., \& Haz, A. M. (2003). Y... iDónde está la resiliencia? Una reflexión conceptual. Revista Interamericana de Psicología, 37(2), 365-372.

Kempe, R. S., \& Kempe, C. H. (1985). Niños maltratados. Madrid: Morata.

Kim, J., \& Cicchetti, D. (2003). Social self-efficacy and behavior problems en maltreated and no maltreated children. Journal of Abnormal Child Psychology, 32(1), 106-117.

Kim, J., \& Cicchetti, D. (2004). A Longitudinal study of child maltreatment, mother-child relationship quality, and maladjustment: The role of self esteem and social competence. Journal of Abnormal Child Psychology, 32(4), 341-354.

Kotliarenco, M. A., Cáceres, I., \& Fontecilla, M. (1997). Estado del arte en resiliencia. Santiago: Organización Panamericana de la Salud, Fundación Ke- llogg, Agencia Sueca de Cooperación Internacional para el Desarrollo.

Lázaro, S. (2009). Resiliencia en niños y adolescentes: revisión teórica e implicaciones para la intervención psicoeducativa en situaciones de maltrato familiar. Estudios de Psicología, 30(1), 89-104.

Luthar, S. (2006). Resilience in development: A synthesis of research across five decades. En D. Cicchetti \& D. J. Cohen (Eds.), Developmental psychopathology. Risk, disorder, and adaptation (Vol. 3, 2.a ed., pp. 739-795). Hoboken, NJ: John Wiley \& Sons.

Luthar, S. S., Cicchetti, D., \& Becker, B. (2000). The construct of resilience: A critical evaluation and guidelines for future work. Child Development, 71(3), 543-562.

Manciaux, M. (2003). La resiliencia: resistir y rehacerse. Barcelona: Gedisa.

Marín, R. (1995). La creatividad: diagnóstico, evaluación e investigación. Madrid: Universidad Nacional de Educación a Distancia.

Martín, E., \& Aciego de Mendoza, R. (2011). Evaluación de un protocolo para valorar situaciones de desprotección infantil: la opinión de los técnicos. Universitas Psychologica, 10(3), 693-704.

Masten, A. (2001). Ordinary magic: Resilience processes in development. American Psychologist, 56(3), 227-238.

Masten, A. S., \& Gewirtz, A. H. (2010). Resiliencia en el desarrollo: la importancia de la primera infancia. En R. E. Tremblay, R. G. Barr, R. De V. Peters \& M. Boivin (Eds.), Enciclopedia sobre el desarrollo de la primera infancia [en línea]. Montreal, Quebec: Centre of Excellence for Early Childhood Development, 1-6. Recuperado de http://www. enciclopedia-infantes.com/documents/MastenGewirtzESPxp.pdf

Mejía, R. (2003). Resiliencia: ilusión o realidad. Revista CES. Medicina, 17(1), 57-62.

Milling, E. (1998). Methodological issues in assessing resilience in maltreated children. Child Abuse and Neglect, 22(7), 669-680.

Melillo, A., \& Suárez Ojeda, N. (2001). Resiliencia. Descubriendo las propias fortalezas. Buenos Aires: Paidós.

Melillo, A., Suárez Ojeda, N., \& Rodríguez, D. (2004). Resiliencia y subjetividad. Buenos Aires: Paidós 
Morelato, G. (2009). Evaluación de la resiliencia en niños víctimas de maltrato familiar (Tesis doctoral inédita). Universidad Nacional de San Luis, San Luis, Argentina.

Morelato, G. (2011a). La resiliencia en el maltrato infantil: aportes para la comprensión del proceso desde un modelo ecológico. Revista de Psicología, 29(2), 203-224.

Morelato, G. (2011b). Maltrato infantil y desarrollo: hacia una revisión de los factores de resiliencia. Pensamiento Psicológico, 9(17), 83-96.

Morelato, G., Maddio, S., \& Ison, M. (2005). Aportes a los criterios de evaluación de las habilidades cognitivas para la solución de problemas interpersonales en una muestra de niños argentinos. Revista Iberoamericana de Diagnóstico y Evaluación Psicológica, 20(2), 149-164.

Moreno, J. (2005, 8 de julio). Maltrato infantil: un estudio sobre la familia, la red de apoyo social y las relaciones de pareja. psiquiatría.com, Artículo 22714. Recuperado de http://www.psiquiatria.com/ articulos/trastornos infantiles/22714

Moreno, J. M. (2006). Revisión de los principales modelos teóricos explicativos del maltrato infantil. Enseñanza e Investigación en Psicología, 11(2), 271-292.

Muela, A. (2008). Hacia un sistema de clasificación nosológico de maltrato infantil. Anales de Psicología, 24(1) 77-87.

Muñoz, A. (2012). El estudio de la resiliencia desde la perspectiva evolutiva y su aportación a la comprensión social del riesgo y la protección en la intervención social. Portularia, 12(1), 9-16.

Naglieri, J., \& LeBuffe, P. (2005). Measuring resilience in children: From theory to practice. En S. Goldstein \& R. Brooks (Eds.), Handbook of resilience in children (pp. 107-124). New York: Kluwer.

Nears, K. (2004). Resilience in maltreated children (Tesis de maestría, Universidad del Estado de Carolina del Norte). Recuperado de http://www.lib.ncsu.edu

Peña, N. (2009). Fuentes de resiliencia en estudiantes de Lima y Arequipa. Liberabit, 15(1), 59-64.

Pollack, W. S. (2006). Sustaining and reframing vulnerability and connection creating genuine resilience in boys and young males. En S. Goldstein \& R. Brooks (Eds.), Handbook of resilience in children (pp. 65-77). New York: Springer.

Rutter, M. (1992). Resilience: Some conceptual considerations. Journal of Adolescent Health, 14(8), 626-631.

Rutter, M. (1995). Resiliencia: algunas consideraciones sobre su concepto. Contemporary Pediatrics, 3(3), 25-38.

Spivack, G., Platt, J., \& Shure, M. (1976). The problem solving approach to adjustment. San Francisco: Jossey-Bass.

Torrance, E. P., \& Miers, R. C. (1970). Desarrollo de la creatividad en el alumno. En R. Marín (Ed.), La creatividad: diagnóstico, evaluación e investigación. Madrid: Universidad Nacional de Educación a Distancia.

Valdez Medina, J. L. (1994). El autoconcepto del mexicano. Estudios de validación. (Tesis doctoral inédita). Universidad Nacional Autónoma de México, México.

Valdez Medina, J. L., Mondragón, J., \& Morelato, G. (2005). El autoconcepto en niños mexicanos y argentinos. Revista Interamericana de Psicología, 39(2), 253-258.

Valdez Medina, J. L., González-Arratia, L. F. N. I., Arantes, J. V., \& Santos, L. A. (1999). El autoconcepto en niños mexicanos y brasileños. Interamerican Journal of Psychology, 33, 205-218.

Valdez-Medina, J. L., González-Arratia, L. F. N. I., Reyes-Lagunes, \& Gil, L. M. (1996). El autoconcepto en niños mexicanos y españoles. Interamerican Journal of Psychology, 30, 179-188.

Vanistendael, S., \& Lecomte, J. (2002). La felicidad es posible. Despertar en niños maltratados la confianza en símismos: construir la resiliencia. Barcelona: Gedisa.

Vecina, M. L. (2006). Creatividad. Papeles del Psicologo, 27(1), 31-39.

Wolin, S., \& Wolin, S. (1993). The resilient self: How survivors of trouble families rise above adversity. Washington: Villard Books. 